13

\title{
Брэгговские дефлекторы волновых потоков для мощных релятивистских мазеров
}

\author{
(C) А.В. Аржанников, ${ }^{1}$ Н.С. Гинзбург, ${ }^{2,3}$ В.Ю. Заславский, ${ }^{2,3}$ П.В. Калинин, ${ }^{1}$ Н.Ю. Песков, ${ }^{2,3,}$ Ф.С. Сергеев, ${ }^{2}$ \\ С.Л. Синицкий
}

${ }^{1}$ Институт ядерной фризики им. Г.И. Будкера СО РАН, 630090 Новосибирск, Россия

${ }^{2}$ Институт прикладной фризики РАН, 603950 Нижний Новгород, Россия

${ }^{3}$ Нижегородский государственный университет им. Н.И. Лобачевского, 603000 Нижний Новгород, Россия

I e-mail: peskov@appl.sci-nnov.ru

Поступило в Редакцию 14 июня 2018 г.

В окончательной редакции 2 октября 2018 г.

Принято к публикации 7 декабря 2018 г.

Исследованы квазиоптические брэгговские структуры, выполненные в виде отрезков планарных волноводов с наклонной (по отношению к направлению распространения волнового пучка) гофрировкой. Показано, что данные структуры являются эффективными поворотными элементами (дефлекторами) для мощных волновых потоков и, таким образом, могут быть использованы для развязки микроволнового излучения и интенсивного электронного потока в релятивистских мазерах. Проведена оптимизация геометрии гофрировки с целью увеличения эффективности трансформации волновых потоков в поперечное направление, а также однородности пространственного распределения рассеянного излучения. Результаты моделирования подтверждены „холодными“ электродинамическими тестами.

DOI: 10.21883/JTF.2019.05.47482.238-18

\section{Введение}

Предложенные в [1,2] брэгговские структуры в виде отрезков волноводов с неглубокой гофрировкой боковых стенок нашли в настоящее время широкое применение в электродинамических системах мощных микроволновых мазеров на основе интенсивных релятивистских электронных пучков (РЭП). В традиционном варианте данные структуры в условиях брэгговского резонанса осуществляют связь и взаимное рассеяние двух встречно-распространяющихся волновых пучков (попутного, синхронно взаимодействующего с РЭП, и встречного, формирующего цикл обратной связи) и, таким образом, выполняют функцию эффективных селективных рефлекторов. Использование резонаторов на основе сочетания различных брэгговских рефлекторов позволило получить устойчивый узкополосный режим генерации в релятивистских мазерах различных типов, включая мазеры на свободных электронах (МСЭ) [2-7], мазеры на циклотронном авторезонансе (МЦАР) [2,810] и черенковские мазеры $[11,12]$.

Теоретическое и экспериментальное исследования планарных схем МСЭ с брэгговскими резонаторами активно ведутся в настоящее время в сотрудничестве ИЯФ СО РАН (Новосибирск) и ИПФ РАН (Н. Новгород) [1316]. Указанная геометрия позволяет также использовать брэгговские структуры с так называемой „наклонной“ гофрировкой для управления направлением распространения рассеянной парциальной волны по отношению к падающей. Таким образом, могут быть реализова- ны дефлекторы волновых потоков. В простейшем виде брэгговский дефлектор представляет собой отрезок планарного волновода с одномерно-периодической гофрировкой, составляющей угол $45^{\circ}$ с направлением поступательного движения электронов и излученного ими волнового пучка, и осуществляет поворот этого волнового пучка на угол $90^{\circ}$ к исходному направлению. Такие дефлекторы хорошо сочетаются с электроннооптическими системами транспортировки сильноточных РЭП и могут быть эффективно использованы в различных планарных схемах МСЭ. В частности, они применимы для развязки мощного выходного излучения и отработанного пучка после пространства взаимодействия [13], в кольцевых резонаторах бегущей волны [14], многопучковых схемах генераторов для передачи волновых потоков между каналами [15] и других устройствах. В настоящей работе представлены результаты исследований электродинамических свойств квазиоптических брэгговских структур планарной геометрии, и определена специфика их применения в качестве дефлекторов волновых потоков в релятивистских мазерах. Результаты теоретического анализа подтверждены трехмерным моделированием и „холодными“ микроволновыми тестами.

\section{1. Принцип работы планарного брэгговского дефлектора и модель связанных волн}

Рассмотрим брэгговский дефлектор, образованный двумя металлическими пластинами ширины $l_{x}$, длины $l_{z}$ 
и расстоянием между ними $a_{0}$, на которые нанесена гофрировка по закону (рис. $1, a)$

$$
a=\frac{a_{1}}{2} \cos \left(\bar{h}_{x} x-\bar{h}_{z} z\right),
$$

где $a_{1}-$ глубина гофрировки, $\bar{h}_{x, z}-$ соответствующие проекции вектора решетки $\bar{\Phi}$ (рис. $1, b), \bar{\Phi}=2 \pi / d, d-$ период гофрировки. Данная решетка обеспечивает связь и взаимное рассеяние двух парциальных волновых потоков, распространяющихся во взаимно-перпендикулярных направлениях:

$$
\mathbf{E}=\mathbf{E}_{A}^{0} \operatorname{Re}\left[A(x ; z) e^{i \omega t-i h_{A} z}\right]
$$

и

$$
\mathbf{E}=\mathbf{E}_{B}^{0} \operatorname{Re}\left[B(x ; z) e^{i \omega t-i h_{b} z}\right],
$$

в условиях брэгговского резонанса

$$
h_{A} \approx \bar{h}_{z}, \quad h_{B} \approx \bar{h}_{x}
$$

Здесь $h_{j}=\sqrt{\omega^{2} / c^{2}-\kappa_{j}^{2}}, \kappa=j \pi / a_{-}-$поперечное волновое число, $j=1,2, \ldots$ - число вариаций поля по поперечной координате $y, A(x, z), B(x, z)$ - медленные функции координат $x$ и $z, \mathbf{E}_{A, B}^{0}(y)$ - поперечные (по оси $y$ ) структуры соответствующих волн невозмущенного планарного волновода:

$$
\mathbf{E}^{0}=i \mathbf{x}_{0} \frac{\omega}{c} \sin \left(\frac{j \pi y}{a_{0}}\right)
$$

для ТЕ-мод и

$$
\mathbf{E}^{0}=\mathbf{z}_{0} \kappa_{j} \sin \left(\frac{j \pi y}{a_{0}}\right)-i \mathbf{y} h_{j} \cos \left(\frac{j \pi y}{a_{0}}\right)
$$

для ТМ-мод, включая основную ТЕМ-моду, которой соответствует $j=0$. Участвующие в процессе рассеяния на гофрировке (1) парциальные волны $A$ и $B$ могут относиться к различным типам волноводных мод и иметь разные постоянные распространения $h_{A} \neq h_{B}$. В простейшем случае, когда брэгговская решетка составляет угол $45^{\circ}$ с каждым из парциальных волновых потоков $\bar{h}_{x, z}=\hbar=\bar{\Phi} / \sqrt{2}$, на ней может быть реализована связь парциальных волн с одинаковыми продольными волновыми числами $h_{A}=h_{B}=h$, и в процессе рассеяния поперечный индекс волн $j$ не меняется. Условие брэгговского резонанса (3) в этом случае имеет вид

$$
h \approx \bar{h} \text {. }
$$

При малой глубине гофрировки $a_{1} \bar{h} \ll 1$ рассеяние парциальных волн $(2, a),(2, b)$ на брэгговской решетке (1) в приближении геометрической оптики может быть описано с помощью уравнения связанных волн для медленно меняющихся амплитуд

$$
\left\{\begin{array}{l}
\frac{\partial A}{\partial z}+i \delta A+i \alpha B=0 \\
\frac{\partial B}{\partial x}+i \delta B+i \alpha A=0
\end{array},\right.
$$

где $\delta=\bar{h}-h$ - отстройка частот волн от точного брэгговского резонанса, $\alpha-$ коэффициент связи волн

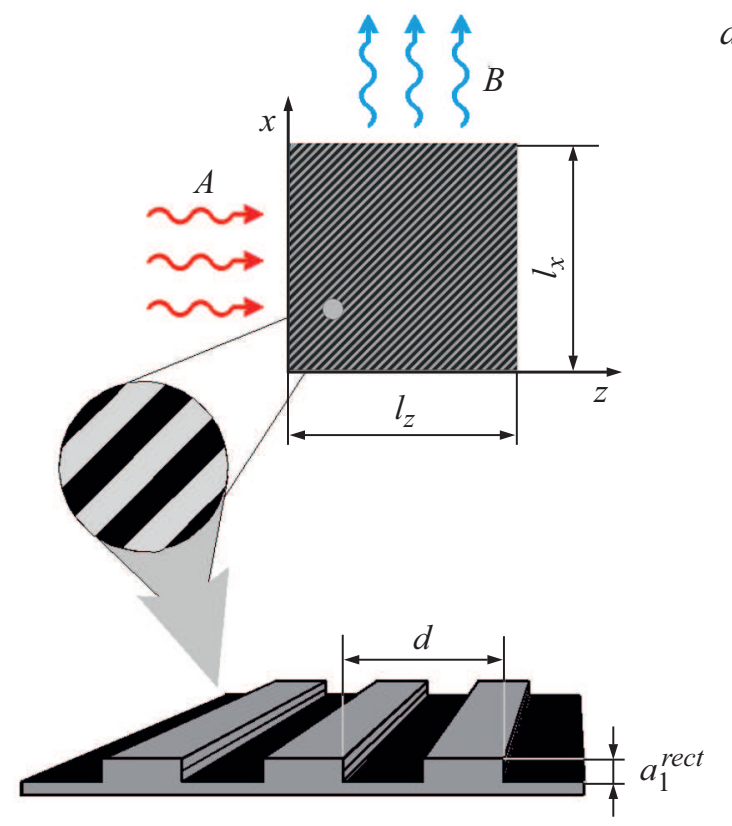

$a$

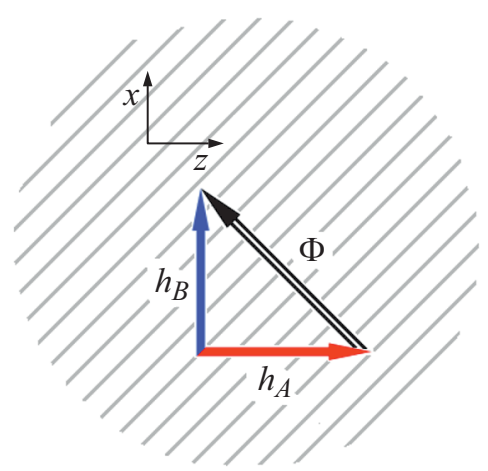

$b$

Pис. 1. Схема планарного дефлектора с гофрировкой в области прямоугольной формы (показана одна из пластин) $(a)$; диаграмма, иллюстрирующая рассеяние парциальных волн на „наклонной“ брэгговской решетке $(b)$.

на двумерной брэгговской структуре, определяемый выражениями (ср. с [17])

$$
\alpha=\frac{a_{1} \bar{h}}{4 a_{0} \varepsilon}
$$

для взаимного рассеяния двух волн ТМ-типа и

$$
\alpha=\frac{\omega a_{1} \kappa}{4 a_{0} c \bar{h} \sqrt{\varepsilon}}
$$

для взаимного рассеяния волны ТМ-типа в волну TE-типа $(\varepsilon=2$ для ТЕМ-волн и $\varepsilon=1$ для остальных мод). Связь двух волн ТЕ-типа на рассматриваемой решетке в первом приближении отсутствует.

Для исследования работы брэгговского дефлектора будем полагать, что при $z=0$ на него падает волновой пучок $A_{0}(x)$, а остальные потоки электромагнитной энергии извне отсутствуют. Это соответствует гранич- 
ным условиям к уравнениям (6) вида

$$
A(x, 0)=A_{0}(x), \quad B(0, z)=0 .
$$

При этом интегральный коэффициент рассеяния излучения в поперечном направлении определяется как

$$
T=\int_{0}^{l_{z}}\left|B_{\text {out }}(z)\right|^{2} d z / \int_{0}^{l_{x}}\left|A_{0}(x)\right|^{2} d x,
$$

где $B_{\text {out }}(z)=B\left(x=l_{x}: z\right)$.

Для теоретического анализа простейшим вариантом задачи является падение плоского однородного волнового пучка, имеющего ТЕМ-структуру по координате $x$, которому соответствует $A_{0}(x)=1$. В данном случае, если область гофрировки представляет собой прямоугольник с размерами $l_{z} \times l_{x}$, решения уравнений (6) на частоте точного брэгговского резонанса даются соотношениями (см. подробнее [17]):

$$
\begin{gathered}
A(x, z)=J_{0}(2 \alpha \sqrt{x z}), \\
B(x, z)=-i \sqrt{\frac{x}{z}} J_{1}(2 \alpha \sqrt{x z}),
\end{gathered}
$$

где $J_{0,1}-$ соответствующие функции Бесселя. Таким образом, в рассматриваемом случае падения плоской ТЕМволны ее полное рассеяние в поперечном направлении имеет место в области, на границе которой $A(x ; z)=0$. Согласно (10), эта область ограничена кривыми

$$
2 \alpha \sqrt{x z}=v_{0, k}
$$

где $v_{0, k}-$ корень функции Бесселя $J_{0}$ с номером $k$. Однако при этом структура волны на выходе дефлектора $B(x ; z)$ оказывается сильно неоднородной по координате $z$, т. е. структура волнового пучка в процессе рассеяния на дефлекторе с простейшей формой гофрировки существенно искажается.

Проведенный далее численный анализ показывает, что оптимизация геометрических размеров области, занятой гофрировкой, позволяет эффективно управлять параметрами волнового пучка на выходе дефлектора, в том числе его поперечной структурой. Результаты моделирования подтверждаются „холодными“ электродинамическими тестами планарных дефлекторов с различной геометрией гофрированной области.

\section{2. Результаты моделирования и электродинамического тестирования планарных брэгговских дефлекторов}

Дальнейший анализ и оптимизация параметров брэгговских дефлекторов ориентированы на их использование в экспериментальных исследованиях планарных

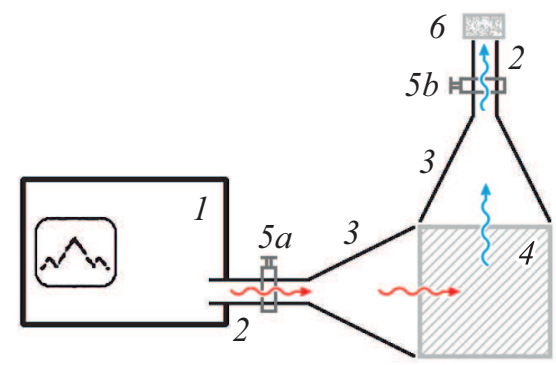

$a$
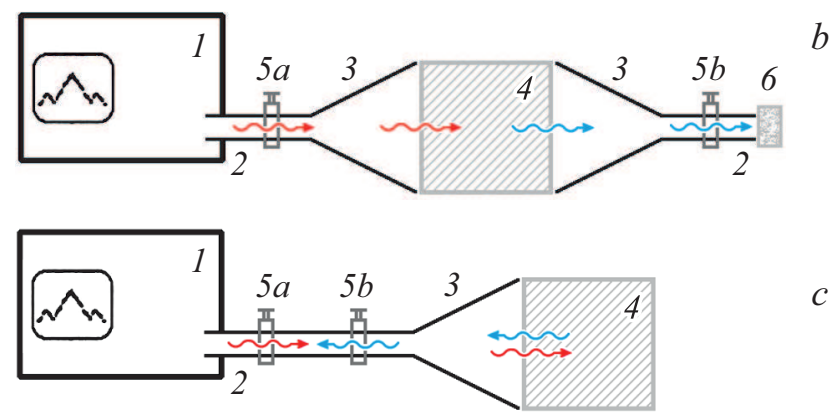

c

Рис. 2. Схема электродинамических экспериментов по измерению коэффициентов поперечного рассеяния $(a)$, прохождения $(b)$ и отражения $(c)$ планарных брэгговских дефлекторов: 1 - панорамный источник, 2 - одномодовые волноводные тракты, 3 - конические волноводные секции, 4 - брэгговский дефлектор, 5, $a$ и $b$ - детекторы падающего и рассеянного волновых пучков соответственно, 6 - поглотитель.

МСЭ на ускорителе „ЭЛМИ“ (ИЯФ СО РАН), в частности, с целью обеспечения вывода излучения из вакуумной системы генератора. Пространство взаимодействия в этих генераторах имеет прямоугольное сечение, сильно вытянутое вдоль одного из поперечных направлений (вдоль оси $x$ в привязке к используемой системе координат). В качестве рабочей волны, взаимодействующей с электронным пучком, используется низшая мода с одной вариацией поля по указанной координате $x^{1}$. Падение данной волны на дефлектор описывается граничным условием

$$
A_{0}=\sin \left(\pi x / l_{x}\right)
$$

Экспериментальное исследование планарных брэгговских дефлекторов проводилось на малом (милливаттном) уровне мощности на специализированном электродинамическом стенде (рис. 2) с использованием панорамных измерителей КСВ Р2-69 (53.5-78.3 GHz) и P2-124 (78.3-118.1 GHz). Запитка брэгговских структур осуществлялась волновым пучком, который формировался при распространении из стандартного волновода по длинной конически расширяющейся секции прямоугольного сечения и имел на выходе этой секции поперечную структуру, близкую к (12). Аналогичная

\footnotetext{
${ }^{1}$ Следует отметить, что номенклатура собственных волн в планарном и прямоугольном волноводах отличается друг от друга. Таким образом, в обсуждаемом случае сильно вытянутого прямоугольного волновода низшей является мода $\mathrm{TE}_{1,0}$, которая при удалении боковых стенок трансформируется в ТЕМ-моду планарного волновода.
} 

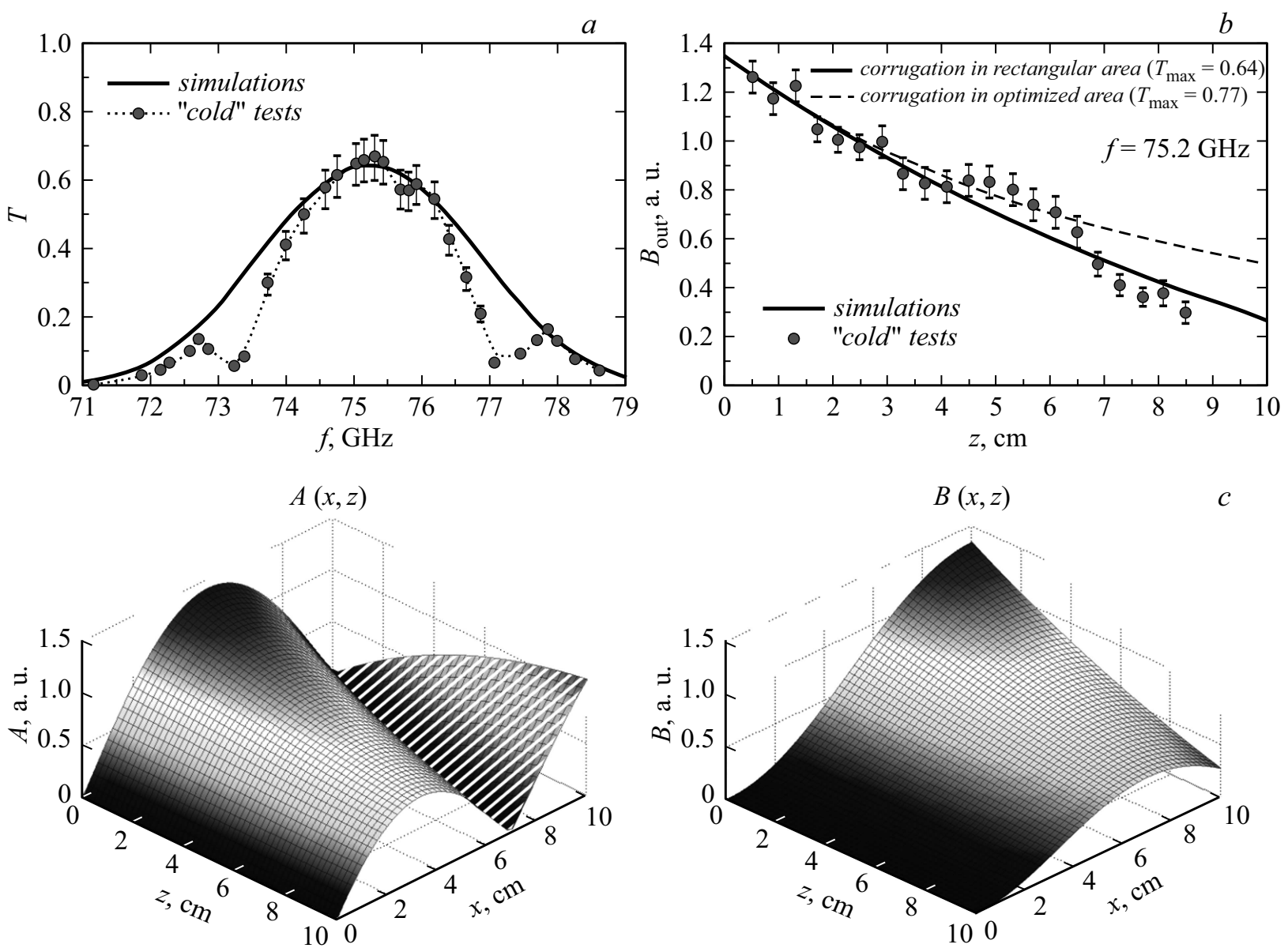

Рис. 3. Результаты моделирования и экспериментального тестирования планарного дефлектора с областью гофрировки прямоугольной формы: $a$ - частотная зависимость коэффициента поперечного рассеяния $T ; b-$ распределение амплитуды парциальной волны на выходе дефлектора в поперечном сечении $B_{\text {out }}(z) ; c$ - пространственная структура полей парциальных волн $A(x, z)$ и $B(x, z)$. Точками на рис. $3, a$ и $b$ показаны результаты “холодных,, электродинамических измерений. Пунктирной кривой на рис. $3, b$ показан результат оптимизации путем удаления части гофрировки, в которой происходит нарастание поля волны $A$ (область штриховки на распределении амплитуды $A(x, z)$ в левой части рис. $3, c)$.

коническая секция использовалась для приема излучения после прохождения дефлектора. В экспериментах измерялся коэффициент рассеяния в поперечном направлении (рис. 2,a), а также контролировались прохождение (рис. 2,b) и отражение (рис. 2,c) от структуры. Калибровка потерь в тракте (и, таким образом, относительного уровня рассеянной на дефлекторе мощности) осуществлялась по схеме, изображенной на рис. 2, $b$, когда вместо брэгговской структуры помещалась секция регулярного планарного волновода соответствующего размера.

В начальных экспериментах были исследованы дефлекторы с наиболее простой геометрией гофрированной области, описанной выше в разд. 1. Эти дефлекторы представляли собой отрезок планарного волновода размером $l_{x}=l_{z}=10 \mathrm{~cm}$, на внутреннюю поверхность пластин которого была нанесена противофазная гофрировка в виде прямоугольных канавок (изображенная на выноске рис. $1, a)$ с периодом $d=2.82 \mathrm{~mm}$ и глубиной $a_{1}^{\text {rect }}=0.3 \mathrm{~mm}$. Очевидно, в случае малой глубины данная гофрировка соответствует „идеальной“ синусоидальной гофрировке (1) с амплитудой основной гармоники $a_{1}^{\text {sin }}=4 a_{1}^{\text {rect }} / \pi$.

Результаты моделирования работы дефлектора с областью гофрировки прямоугольной (в данном случае квадратной) формы приведены на рис. 3. Здесь представлены частотные зависимости коэффициента рассеяния излучения в поперечном направлении $T(f), f=\omega / 2 \pi$, полученные в результате численного решения уравнений (6) с граничными условиями (8), (12). Согласно результатам моделирования, указанный дефлектор обеспечивает достаточно высокую эффективность (до $\sim 65 \%$ по мощности) трансформации излучения в окрестности рабочей частоты $75 \mathrm{GHz}$ (рис. 3,a). Результаты „холодных“ измерений данного дефлектора, представленные на рис. $3, a$ и $b$, демонстрируют хорошее соответствие с моделированием, и соответственно адекватность теоретической модели на основе метода связанных волн. Ширина полосы частот, в которой реализуется поворот потока излучения, составляет около $2-2.5 \mathrm{GHz}$, что зна- 

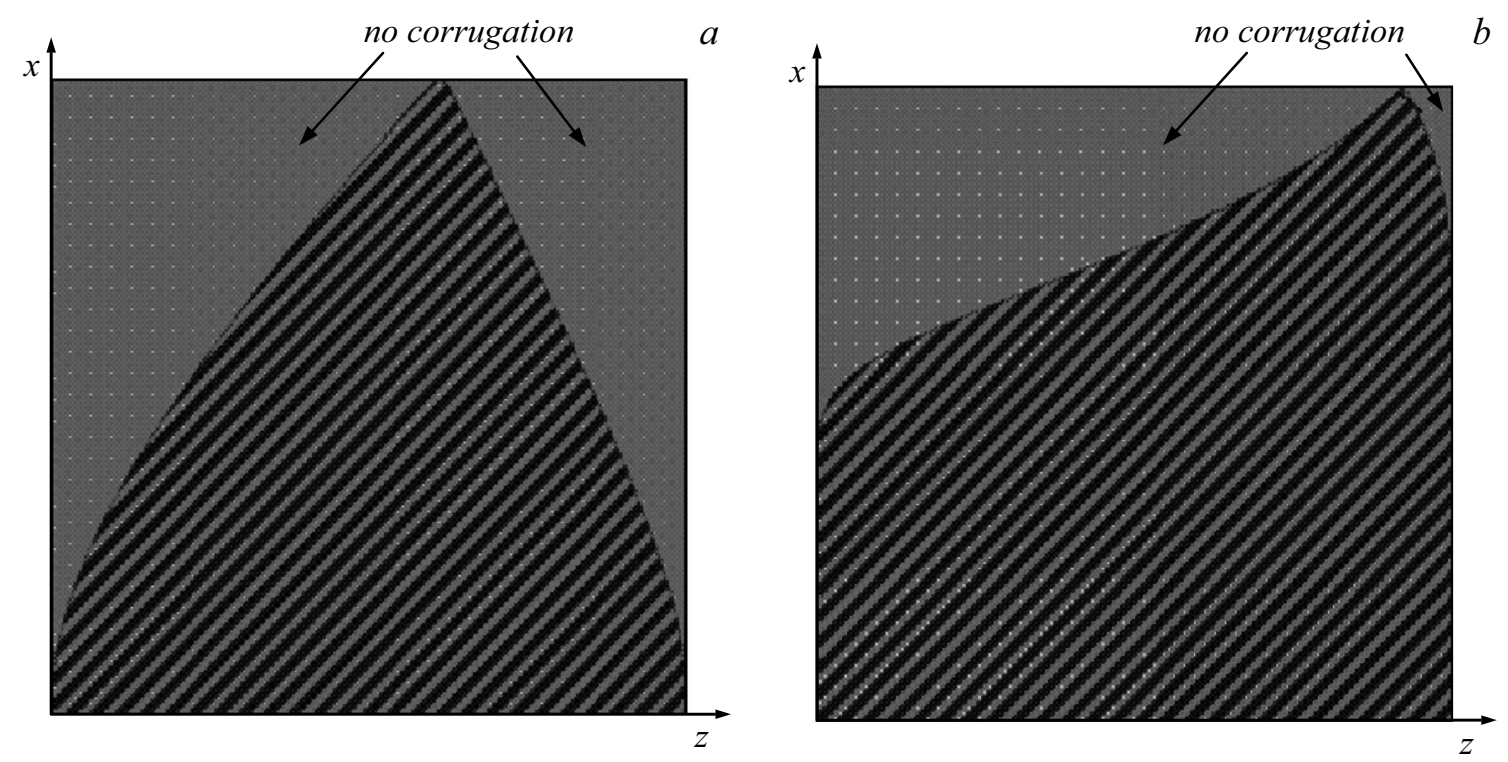

Рис. 4. Форма областей гофрировки планарных дефлекторов, обеспечивающих на выходе формирование волновых потоков с синусоидальной $(a)$ и квазиоднородной $(b)$ поперечной структурой (вдоль координаты $x$ ).

чительно превышает рабочую частотную полосу резонаторов, которые использовались в экспериментальных реализациях планарных МСЭ. Это позволяет обеспечить вывод излучения с приемлемой эффективностью. В то же время анализ поперечного распределения амплитуды волнового пучка на выходе дефлектора $B_{\text {out }}(z)$ при $x=10 \mathrm{~cm}$ (рис. $3, b)$ показывает, что на дефлекторе с прямоугольной областью гофрировки возникает сильное искажение структуры поля, что затрудняет его последующее согласование с выходным волноводным трактом на основе волноводов прямоугольного сечения.

Как уже отмечалось выше, оптимизация геометрии области гофрировки дефлектора позволяет управлять параметрами выходного волнового пучка. На рис. $3, c$ представлено пространственное распределение амплитуд парциальных волн $A(x ; z)$ и $B(x ; z)$ внутри дефлектора на „центральной“ частоте $75.2 \mathrm{GHz}$, соответствующей точному брэгговскому резонансу в (5). Видно (левая половина рис. 3,c), что внутри дефлектора существует область, в которой амплитуда падающей парциальной волны $A$ нарастает вдоль его выходной границы (данная область на поверхности значений функции $A(x ; z)$ помечена штриховкой). Этим объясняется понижение коэффициента трансформации в волну $B$ в этой пространственной области дефлектора (правая половина рис. $3, c)$. Отсюда следует, что эффективность дефлектора может быть повышена путем удаления гофрировки в этой его области. Согласно проведенным расчетам, такое изменение геометрии гофрированной поверхности позволяет увеличить долю выводимой мощности до уровня $75-80 \%$, однако оно не решает задачу согласования рассеянной волны и выходного волноводного тракта (штриховая кривая на рис. $3, b$ ).

\section{3. Оптимизация формы гофрированной области с целью управления пространственной структурой выходного излучения}

Наиболее важным, с точки зрения обсуждаемых приложений, является формирование требуемой структуры рассеянной парциальной волны на выходе дефлектора, которое может быть достигнуто путем оптимизации геометрии гофрированной области. Здесь мы приведем два примера формы областей гофрировки пластин, которые применимы к получению синусоидального (рис. $4, a)$ и квазиоднородного (рис. $4, b)$ распределений плотности мощности по сечению выходного волнового потока. Синусоидальное распределение необходимо для согласования с системами вывода на основе закрытых прямоугольных сверхразмерных волноводов, а квазиоднородное требуется для согласования с планарными открытыми системами, использующими гауссовы пучки. На рис. 5 показаны результаты моделирования распределения поля рассеянной волны на выходе дефлектора на центральной частоте $75.2 \mathrm{GHz}$ при вариации формы гофрированной поверхности и глубины гофрировки (с указанием максимального интегрального коэффициента поперечного рассеяния $T_{\max }$ ). Расчеты показывают, что при увеличении глубины гофрировки до $0.4 \mathrm{~mm}$ может быть достигнута высокая эффективность трансформации (до $80-85 \%$ по мощности) в волновой пучок с заданной поперечной структурой. Максимальная эффективность трансформации (без дополнительного условия на однородность поперечного распределения) при такой глубине гофрировки достигает более $90 \%$.

Для экспериментального тестирования был спроектирован и изготовлен оптимизированный дефлектор 

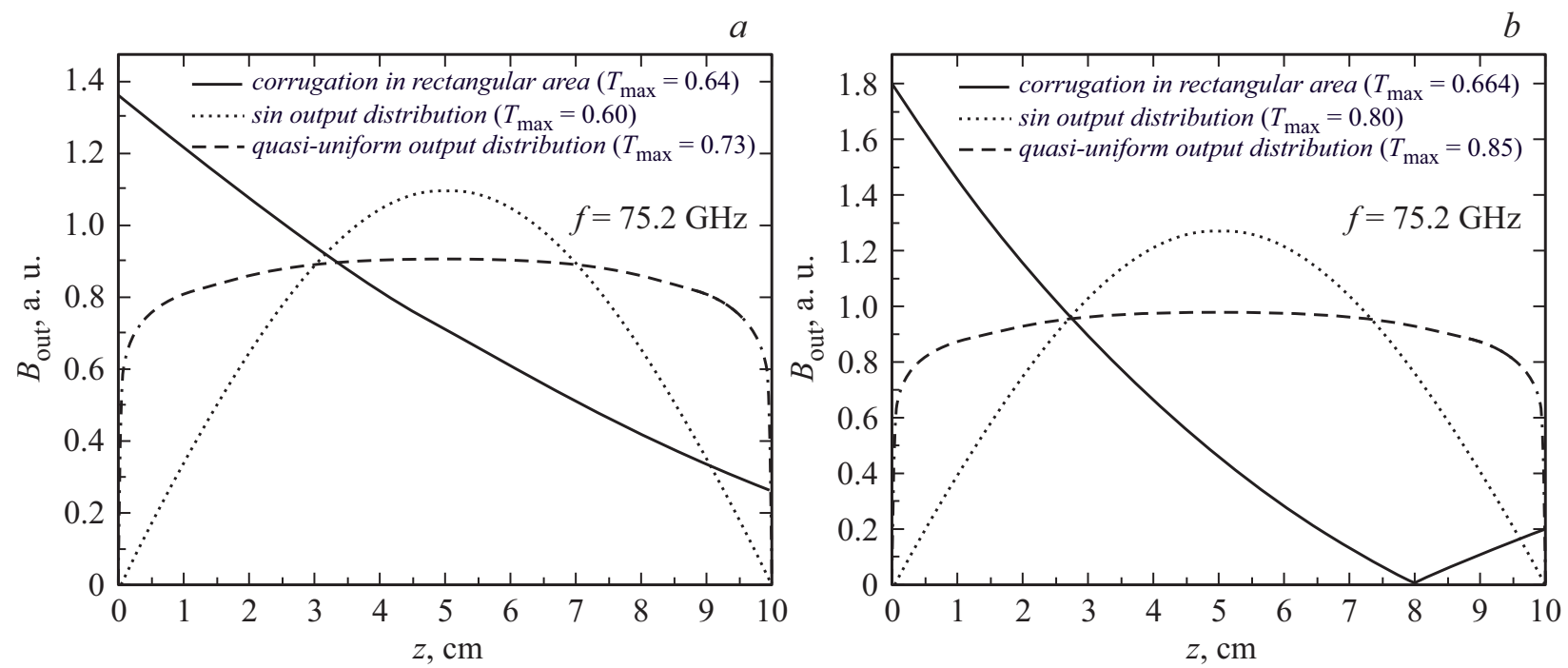

Рис. 5. Результаты моделирования электродинамических характеристик планарных брэгговских дефлекторов с различной геометрией гофрированной области. Распределения амплитуды парциальной волны $B_{\text {out }}(z)$ на выходе дефлектора на центральной частоте $75.2 \mathrm{GHz}$ при глубине гофрировки $a_{1}^{\text {rect }}=0.3 \mathrm{~mm}(a)$ и $0.4 \mathrm{~mm}(b)$. В подписях приведена интегральная мощность поперечного рассеяния $T_{\max }$ на указанной частоте.
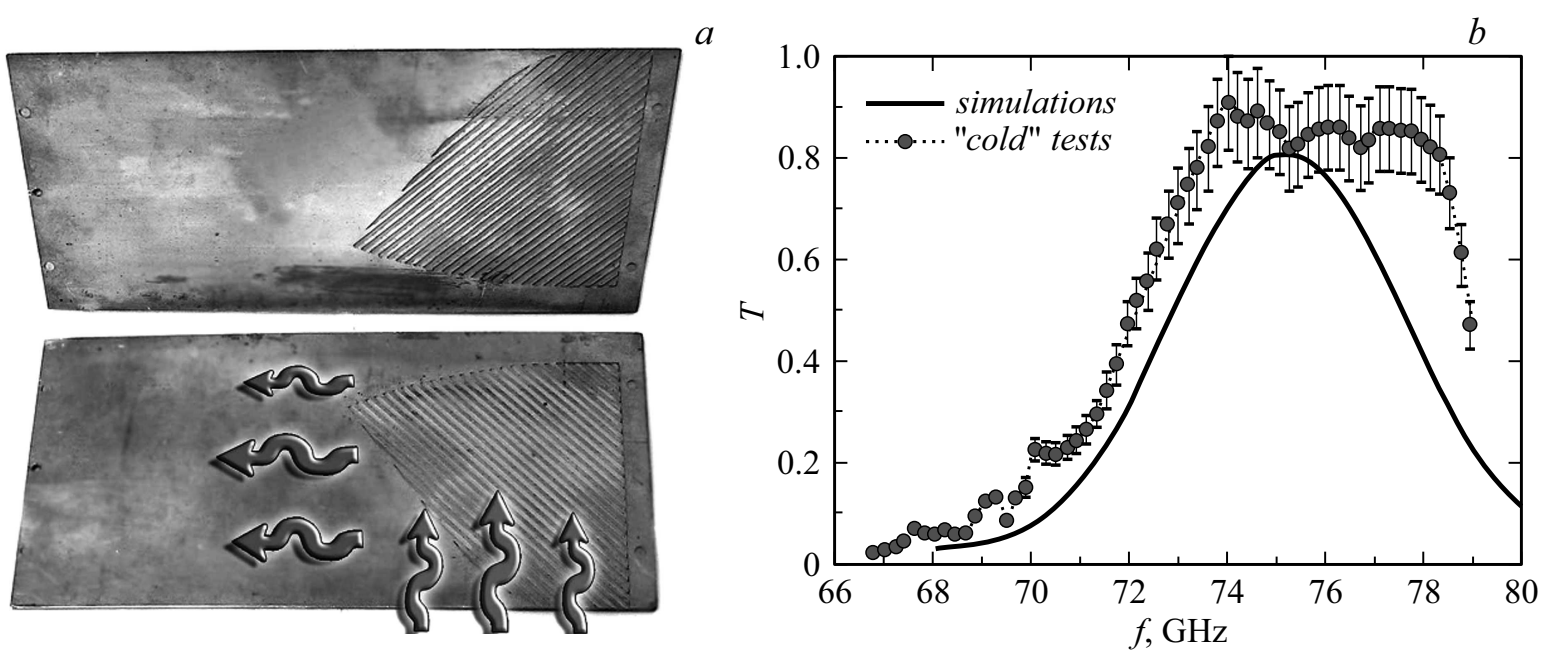

Рис. 6. Результаты экспериментального исследования оптимизированного планарного дефлектора: $a-$ фотография гофрированных пластин (показаны направления падающего и рассеянного волновых потоков) и $b$ - частотная зависимость коэффициента поперечной трансформации мощности $T$ (сплошная кривая - результаты моделирования, точки - результаты измерений).

(рис. 6,a), формирующий синусоидальное распределение поля, что обеспечивает согласование дефлектора с выходным трактом планарного МСЭ. Для достижения высокого коэффициента трансформации глубина гофрировки составляла $0.4 \mathrm{~mm}$. В соответствии с результатами моделирования в „холодном“ тестировании указанного дефлектора был получен коэффициент трансформация по мощности $\sim 80 \%$ (рис. $6, b$ ) и близкое к синусоидальному распределение поля рассеянной волны. Измеренная полоса частот брэгговского рассеяния составила 74-78 GHz, что несколько превосходит расчетную полосу, полученную в результате моделирования на основе метода связанных волн. Увеличение глубины гофри- ровки приводит к нарушению условия применимости одномодового (по оси $y$ ) приближения, использованного при записи уравнений (6), и требует применения более полных методов анализа. Результаты трехмерного моделирования описываемого дефлектора с использованием кода CST Microwave Studio представлены на рис. 7. Эти результаты хорошо согласуются с результатами экспериментального тестирования как по структуре поля, так и по полосе частот.

Следует, однако, отметить, что увеличение ширины рабочей частотной полосы дефлектора может быть нежелательно в ряде приложений, в частности, для использования дефлекторов в МСЭ на основе кольцевого 

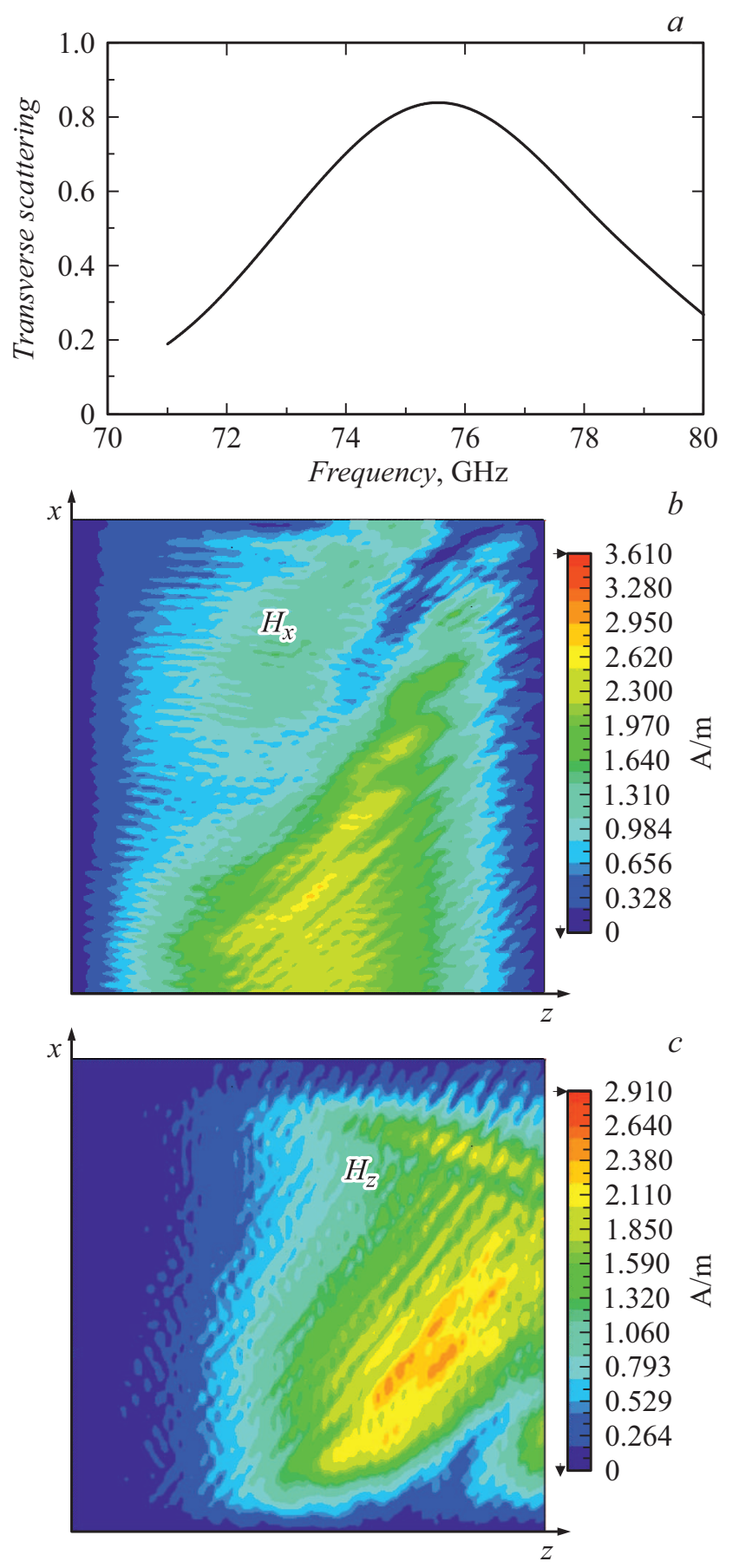

Рис. 7. Результаты трехмерного моделирования оптимизированного планарного дефлектора на основе кода CST Microwave Studio: $a$ - частотная зависимость коэффициента поперечной трансформации мощности и $b-$ пространственная структура компонент магнитного поля $\left(H_{x}\right.$ соответствует парциальной волне $A, H_{z}$ - парциальной волне $\left.B\right)$.

резонатора бегущей волны, составленного из указанных элементов [14]. В этом случае для увеличения селективности возбуждения рабочего типа колебаний требуется более узкая частотная полоса. Сохранить узкую частотную полосу в сочетании с высоким коэффициентом по- перечного рассеяния можно путем увеличения размеров гофрированной области дефлектора при относительно небольшой глубине гофрировки. С точки зрения анализа это позволяет оставаться в рамках применимости модели связанных волн (т. е. уравнений (6)).

В рамках этого подхода был исследован дефлектор с размером $l_{x}=l_{z}=19 \mathrm{~cm}$ с гофрировкой поверхно-

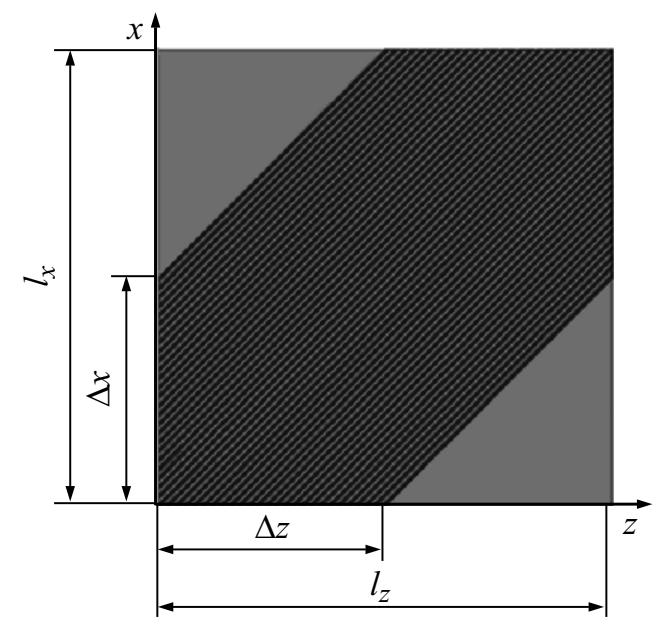

$a$
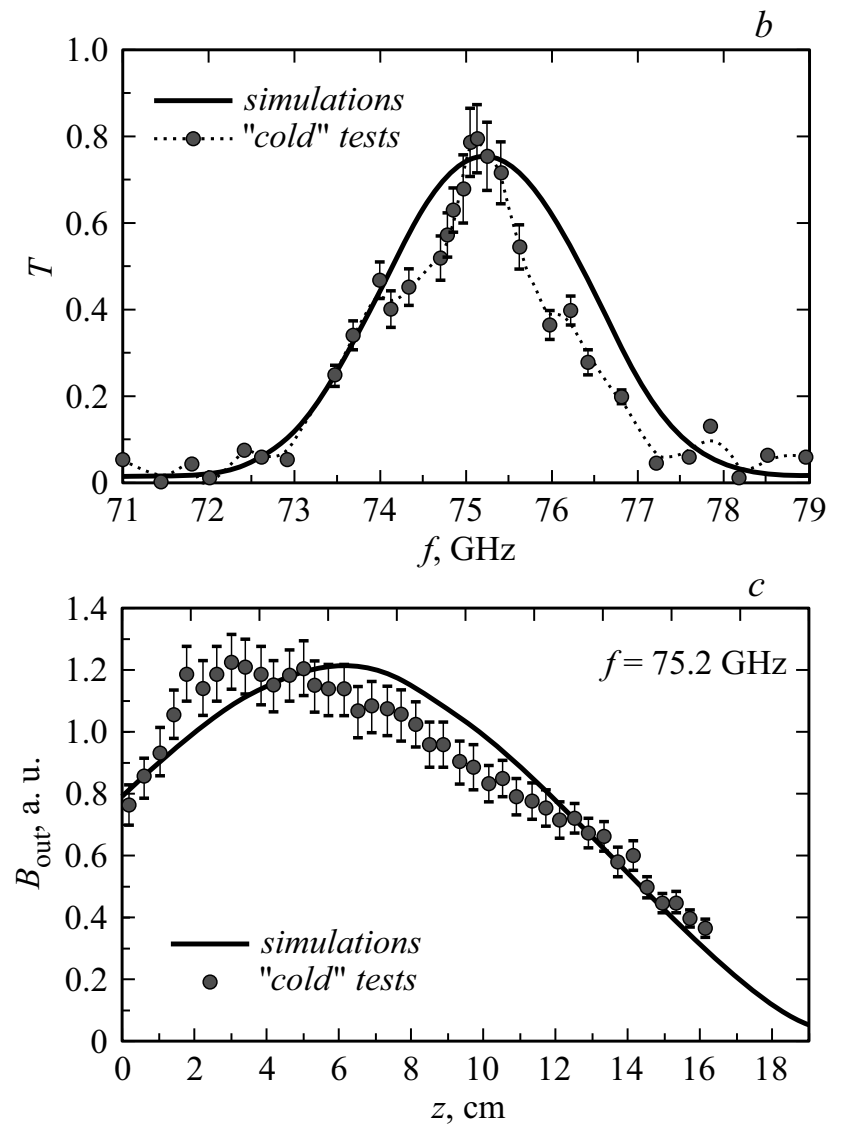

Рис. 8. Планарный дефлектор с областью гофрировки в виде „диагональной полосы“: $a-$ схема гофрировки, $b-$ частотная зависимость коэффициента поперечного рассеяния $T, c-$ распределение амплитуды рассеянной парциальной волны на выходе дефлектора $B_{\text {out }}(z)$ на центральной частоте $75.2 \mathrm{GHz}$ (сплошная кривая - расчет, точки - результаты измерений). 
сти в виде полосы, расположенной симметрично относительно диагонали квадрата и ограниченной линиями, сдвинутыми от диагонали на $\Delta_{x}=\Delta_{z}=10 \mathrm{~cm}$ (рис. 8,a). В данном случае глубина гофрировки составляла $a_{1}^{\text {rect }}=0.2 \mathrm{~mm}$ Результаты моделирования и холодных измерений характеристик этого дефлектора сопоставлены на рис. $8, b$ и $c$. Коэффициент трансформации мощности в волновой поток, распространяющийся в поперечном направлении (рис. $8, b$ ), составляет до 80\% при относительно узкой полосе частот $\sim 2-2.5 \mathrm{GHz}$ (по уровню $-3 \mathrm{~dB}$ ), что обеспечено малой глубиной гофрировки. В то же время моделирование показывает, что увеличение глубины гофрировки в указанной пространственной области до $0.3 \mathrm{~mm}$ позволяет повысить коэффициент рассеяния в поперечном направлении $T$ до 95\% при расширении частотной полосы до $3-3.5 \mathrm{GHz}$. Принципиальным достоинством дефлектора данного вида является сохранение поперечной структуры парциальных волновых потоков. Как видно из рис. $8, c$, рассеянный в дефлекторе волновой поток имеет синусоидальное поперечное распределение, аналогичное падающему потоку. Вместе с тем определенным недостатком дефлектора c реализованной конфигурацией гофрировки является наличие смещения волнового пучка от геометрического центра дефлектора, которое имеет как в моделировании, так и в тестовом эксперименте величину около 4-5 cm. Данное смещение может быть скомпенсировано сдвигом гофрированной полосы вдоль оси $z$ за счет изменения величин $\Delta_{x}$ и $\Delta z$ при сохранении остальных геометрических размеров дефлектора.

\section{4. Обсуждение результатов и выводы}

Таким образом, проведенные исследования демонстрируем высокую эффективность предложенной схемы дефлекторов на основе квазиоптических планарных брэгговских структур с наклонной (по отношению к направлению распространения парциальных волн) гофрировкой для рассеяния мощных волновых потоков в поперечное направление. Для теоретического анализа указанных схем развит усредненный подход, основанный на методе связанных волн. Показано, что оптимизация геометрии области, занятой гофрировкой, позволяет обеспечить высокий коэффициент рассеяния парциальных волн, а также формировать поперечную структуру выходного излучения, необходимую для дальнейшего согласования дефлектора с различными электродинамическими системами. Результаты теоретического анализа подтверждены экспериментальным тестированием брэгговских дефлекторов с различной геометрией гофрированной области.

Следует отметить, что в моделировании и экспериментальных тестах падающие и рассеянные волновые пучки имели одинаковую поперечную структуру (по оси $y$ ) и соответственно абсолютные значения продольных волновых чисел. В то же время изменение угла гофрировки позволяет осуществлять связь мод различного типа, т. е. рассеяние излучения в поперечное направление может сопровождаться изменением индекса волны по оси $y$ (т.е. индекса $j$, выражение (4) в разд. 1). Рассеяние в волны с высоким индексом привлекательно с точки зрения увеличения коэффициента связи на брэгговской гофрировке и повышения эффективности дефлектора.

Важным достоинством брэгговских дефлекторов является хорошая совместимость с магнитными и электронно-оптическими системами мощных планарных МСЭ на основе сильноточных ленточных РЭП. Указанные дефлекторы были успешно использованы в экспериментальной реализации $75 \mathrm{GHz}$ МСЭ на ускорителе „ЭЛМИ“ (1 MeV/5 kA/5 $\mu$ s) [13]. В данном эксперименте установленный на выходе пространства взаимодействия дефлектор позволил выполнить развязку рабочей волны с отработанным сильноточным РЭП, транспортируемым к коллектору, путем ее поворота в поперечное (по отношению к поступательной скорости пучка) направление и, таким образом, осуществить вывод излучения мультимегаваттного уровня мощности в свободное пространство через выходное окно. Проведенные в [13] измерения выходного излучения находились в хорошем соответствии с результатами моделирования как по уровню мощности, так и по частоте и структуре (диаграмме направленности) выходного излучения и, таким образом, демонстрировали в том числе работоспособность выходной секции на основе разработанных брэгговских дефлекторов в условиях высокой интенсивности волновых потоков.

Системы из двух и более дефлекторов позволяют реализовать многоканальные схемы планарных генераторов $[14,15]$. Одним из вариантов подобных компоновок является рассмотренный в [14] кольцевой резонатор бегущей волны, образованный последовательно установленными четырьмя планарными брэгговскими дефлекторами. Согласно проведенному анализу [14], использование подобного резонатора позволяет реализовать двухстадийную схему МСЭ на основе двух параллельных сильноточных ленточных РЭП, один из которых запитывает генератор волны накачки, а на втором пучке реализуется процесс вынужденного встречного рассеяния с преобразованием частоты из миллиметрового в терагерцовый диапазоны. Исследования двухстадийных МСЭ проводятся в настоящее время на экспериментальном комплексе „ЭЛМИ“, в качестве ключевых элементов электродинамических систем генераторов данного типа используются описанные выше схемы брэгговских дефлекторов [18].

Кроме электродинамических систем МСЭ применение квазиоптических брэгговских дефлекторов представляется перспективным также в качестве полосовых фильтров и мультиплексеров для анализа широкополосных сигналов. Наклонная гофрировка позволяет вырезать из исследуемого сигнала импульс в определенной частотной области и ответвить его в поперечном направлении для дальнейшего преобразования, а последова- 
тельность таких фильтров - распределить исследуемый сигнал по калиброванному набору частотных полос. При этом шириной полос пропускания таких фильтров можно управлять изменением глубины гофрировки. По сравнению с диэлектрическими аналогами, исследованными в [19], предлагаемые системы обладают существенно большей электропрочностью и, как показал опыт их использования в МСЭ, дают возможность работать с микроволновым излучением высокой мощности, вплоть до мультимегаваттного уровня.

Авторы признательны Г.Г. Денисову и В.И. Белоусову за полезные обсуждения. Работа выполнена при частичной поддержке РФФИ (грант № 16-08-00811).

\section{Список литературы}

[1] Ковалев Н.Ф., Орлова И.М., Петелин М.И. // Изв. вузов. Радиофизика. 1968. Т. 11. Вып. 5. С. 783-786. [Kovalev N.F., Orlova I.M., Petelin M.I. // Radiophys. Quant. Electron. 1972. Vol. 11. N 5. P. 449-450.]

[2] Bratman V.L., Denisov G.G., Ginzburg N.S., Petelin M.I. // IEEE J. Quantum. Elect. 1983. Vol. QE-19. N 3. P. 282-296.

[3] Ciocci F., Bartolini R., Doria A., Gallerano G.P. et al. // Phys. Rev. Lett. 1993. Vol. 70. P. 928-931.

[4] Chu T.S., Hartemann F.V., Danly B.G., Temkin R.J. // Phys. Rev. Lett. 1994. Vol. 72. N 15. P. 2391-2395.

[5] Cross A.W., Ginzburg N.S., He W. et al. // Nucl. Instrum. Meth. A. 1998. Vol. 407. P. 181-186.

[6] Песков Н.Ю., Гинзбург Н.С., Каминский А.А. и др. // Письма в ЖТФ. 1999. Т. 25. Вып. 11. С. 19-29. [Peskov N.Yu., Ginzburg N.S., Kaminsky A.A. et al. // Tech. Phys. Lett. 1999. Vol. 25. N 6. P. 429-432.]

[7] Каминский А.К., Перельштейн Э.А., Седых С.Н. и др. // Письма в ЖТФ. 2010. Т. 36. Вып. 5. С. 37-46. [Kaminsky A.K., Perelshtein E.A., Sedykh S.N. et al. // Tech. Phys. Lett. 2010. Vol. 36. N 3. P. 211-215.]

[8] Fliflet A.W., McCowan R.B., Sullivan C.A. et al. // Nucl. Instrum. Meth. A. 1989. Vol. 285. P. 233-238.

[9] Alberti S., Danly B.G., Gulotta G. et al. // Phys. Rev. Lett. 1993. Vol. 71. N 13. P. 2018-2021.

[10] Bratman V.L., Denisov G.G., Kol'chugin B.D. et al. // Phys. Rev. Lett. 1995. Vol. 75. N 17. P. 3102-3105.

[11] Абубакиров Э.Б., Денисенко А.Н., Савельев А.В. и др. // Письма в ЖТФ. 2000. Т. 26. Вып. 4. С. 14-18. [Abubakirov E.B., Denisenko A.N., Savel'ev A.V. et al. // Tech. Phys. Lett. 2000. Vol. 26. N 2. P. 141-142.]

[12] Benford J., Swegle J., Shamiloglu E. High Power Microwaves. 3-rd edition. CRC Press, 2015. $470 \mathrm{c}$.

[13] Аржсанников А.В., Гинзбург Н.С., Заславский В.Ю. и др. // Письма в ЖЭТФ. 2008. Т. 87. Вып. 11. С. 715-719. [Arzhannikov A.V., Ivanenko V.G., Ivanov I.A. et al. // JETP Lett. 2008. Vol. 87. N 11. P. 618-622.]

[14] Аржсанников А.В., Гинзбург Н.С., Денисов Г.Г. и др. // Письма в ЖТФ. 2014. Т. 40. Вып. 17. С. 11-21. [Arzhannikov A.V., Ginzburg N.S., Denisov G.G. et al. // Tech. Phys. Lett. 2014. Vol. 40. N 9. P. 730-734.]

[15] Аржсанников А.В., Гинзбург Н.С., Заславский В.Ю. и др.// Вестник НГУ. Физика. 2012. Т. 1. Вып. 7. С. 5-14.
[16] Arzhannikov A.V., Ginzburg N.S., Kalinin P.V. et al. // Phys. Rev. Lett. 2016. Vol. 117. N 11. P. 114801.

[17] Гинзбург Н.С., Песков Н.Ю., Сергеев А.С. // Радиотехника и электроника. 1995. Т. 40. Вып. 3. С. 401-414. [Ginzburg N.S., Peskov N.Yu., Sergeev A.S. // J. Commun. Technol. Electron. 1995. Vol. 40. N 5. P. 8-21.]

[18] Arzhannikov A.V., Ginzburg N.S., Kalinin P.V. et al. // EPJ Web of Conferences. 2017. Vol. 149. P. 05017.

[19] Белоусов В.И., Вершков В.А., Денисов Г.Г. и др. // Письма в ЖТФ. 2017. Т. 43. Вып. 22. С. 83-90. [Belousov V.I., Vershkov V.A., Denisov G.G. et al. // Tech. Phys. Lett. 2017. Vol. 43. N 11. P. 1037-1040.] 